\title{
Inflammatory pseudotumors of the kidney and the lung presenting as immunoglobulin G4- related disease: a case report
}

Genya Nishikawa*, Kogenta Nakamura, Yoshiaki Yamada, Takahiko Yoshizawa, Yoshiharu Kato, Remi Katsuda, Kenji Zennami, Motoi Tobiume, Shigeyuki Aoki, Tomohiro Taki and Nobuaki Honda

\begin{abstract}
Introduction: It has been reported that immunoglobulin G4-related systemic disease can spread to nearly every organ, and often presents as an inflammatory mass or masses at those sites. In the kidney, this disease is often diagnosed after a radical or partial nephrectomy following the discovery of an inflammatory mass which is often suspected to be a malignant tumor. Here, we present a rare case of inflammatory pseudotumors of the kidney and the lung presenting as immunoglobulin G4-related disease, which were diagnosed by computed tomographyguided biopsies.
\end{abstract}

Case presentation: A 54-year-old Japanese man was referred to our hospital with suspected bilateral renal cancer, multiple lung metastases and autoimmune pancreatitis. His serum immunoglobulin G4 level was high. We used computed tomography-guided biopsies and histopathological examinations of the biopsied specimens to diagnose the tumors as immunoglobulin G4-related bilateral renal and lung inflammatory pseudotumors. Our patient was treated with oral prednisolone, and after one month of treatment, contrast-enhanced computed tomography demonstrated a general improvement, as noted by a reduction in size of the masses.

Conclusion: Renal masses that are formed due to immunoglobulin G4-related disease require comprehensive diagnosis to prevent unnecessary surgical resections from being performed. Further consideration should be paid to immunoglobulin G4-related diseases in the future.

\section{Introduction}

Patients with autoimmune pancreatitis often exhibit high serum immunoglobulin G4 (IgG4) levels, and/or marked infiltration of IgG4-positive plasma cells, both of which are hallmarks of IgG4-related systemic disease. In addition to the pancreas, IgG4-related mass-forming lesions have also been described in other organs. In the kidney, IgG4-related disease can also present as an inflammatory mass, and is often diagnosed after a radical or partial nephrectomy due to suspected malignancy. Here, we present a case report of a patient with IgG4related bilateral renal and lung inflammatory pseudotumors. We also describe the diagnostic process and treatment course.

\footnotetext{
* Correspondence: genchari@aichi-med-u.ac.jp

* Correspondence: genchari@aichi-med-u.ac.jp
Department of Urology, Aichi Medical University School of Medicine, Nagakute, Aichi 480-1195, Japan
}

\section{Case presentation}

A 54-year-old Japanese man presented to a Department of Internal Medicine with chief complaints of dry mouth and weight loss that he had been experiencing for two months. He had type 1 diabetes mellitus, which was being treated with insulin. Computed tomography (CT) scans showed the presence of bilateral renal and pulmonary masses, and swelling of his pancreatic parenchyma. He was referred to our hospital with suspected bilateral renal cancer, multiple lung metastases and autoimmune pancreatitis. He had bronchial asthma, but no habitual contributory factors. Blood tests revealed high IgG $(1775 \mathrm{mg} / \mathrm{dL})$ and IgG4 levels $(351 \mathrm{mg} / \mathrm{dL})$. Lactate dehydrogenase and C-reactive protein levels, which are prognostic factors for renal cancer, were nor$\mathrm{mal}(162 \mathrm{U} / \mathrm{L}$ and $0 . \mathrm{mg} / \mathrm{dL}$, respectively). No other abnormal values were noted, including urine analysis results. Contrast-enhanced CT scans showed multiple 
nodular opacities of various sizes with irregular margins in both lung fields. In his abdomen, there were slight poorly enhanced mass lesions (left, $10 \times 10 \mathrm{~mm}$; right, $18 \times 14 \mathrm{~mm}$ ) in the upper pole of both kidneys (Figure 1). Magnetic resonance imaging showed a mass in each kidney with a low signal intensity on the T1- and T2weighted images, and poorly enhanced areas inside each mass. Magnetic resonance cholangiopancreatography and endoscopic retrograde cholangiopancreatography were performed, and our patient was diagnosed with autoimmune pancreatitis. The bilateral renal and pulmonary masses were suspected of being multiple lung metastases stemming from bilateral renal cancer, but inflammatory pseudotumors associated with autoimmune pancreatitis could not be ruled out. Based on these findings, a CT-guided biopsy was performed on the right pulmonary mass and right renal mass. Histopathological examination of the biopsy specimens showed extensive fibrous tissue around the glomeruli in his kidney and alveoli in his lung. Infiltration of lymphocytes and plasma cells was also observed. A malignant tumor was considered unlikely because no atypical cells were observed. However, immunohistochemical staining revealed the presence of IgG- and IgG4-positive plasma cells; the number of IgG4-positive plasma cells was 44 cells per high power field (HPF) as shown in Figure 2. Based on these findings, we diagnosed the tumor as IgG4-related bilateral renal and multiple pulmonary masses.

Following diagnosis, treatment with oral prednisolone (40 mg/day) was initiated. A CT scan performed on the ninth day of treatment showed a reduction in the size of the masses, so the dose was decreased to $30 \mathrm{mg}$. Subsequent $\mathrm{CT}$ scans showed a further reduction in the size

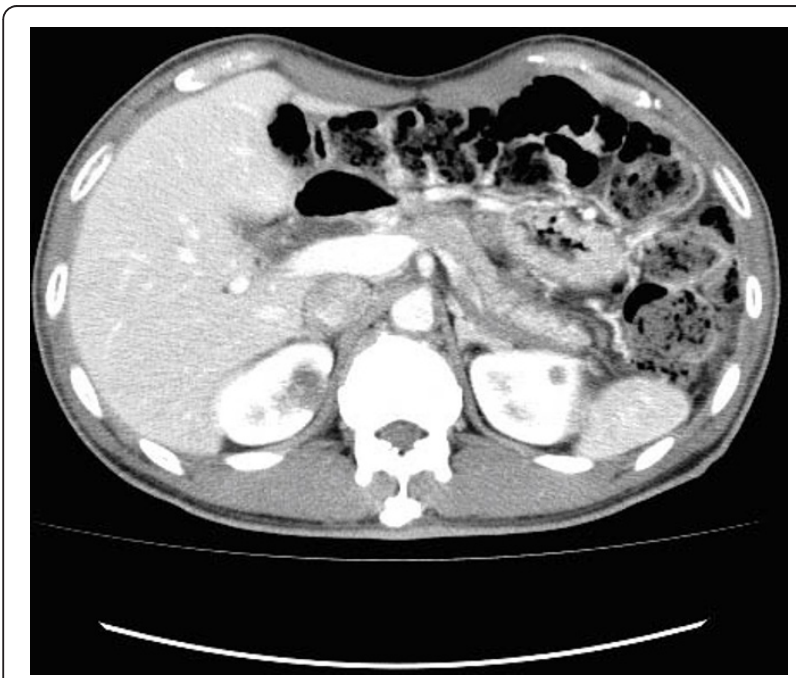

Figure $1 \mathrm{CT}$ showed poorly enhanced mass lesions (left, $10 \times$ $10 \mathrm{~mm}$; right, $18 \times 14 \mathrm{~mm}$ ) in the upper part of both kidneys.

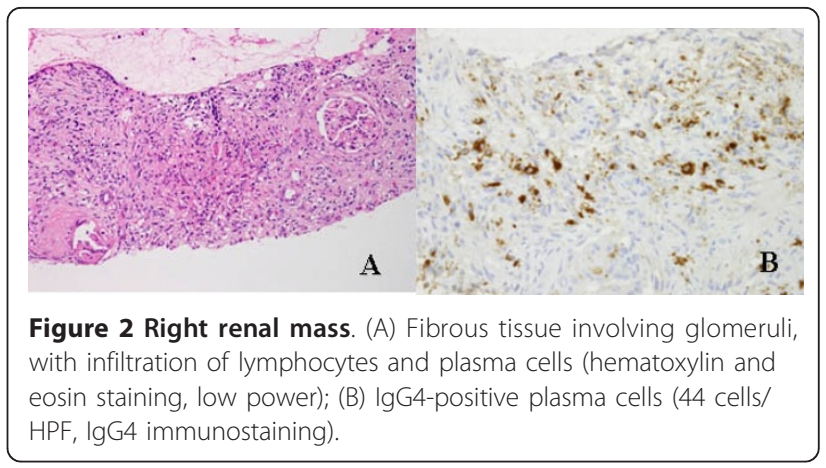

of the masses so treatment with prednisolone was tapered. After one month of treatment, contrastenhanced CT revealed new, small pulmonary lesions, but also demonstrated a general improvement, with a reduction in size of the right renal mass, and disappearance of the left renal mass (Figure 3). Serum IgG levels decreased to values within the normal range after initiation of treatment with prednisolone. Our patient continued receiving $10 \mathrm{mg}$ prednisolone per day to prevent a recurrence, which to date, has not occurred.

\section{Discussion}

In 2001, Hamano et al. first reported that patients with autoimmune pancreatitis have high serum IgG4 levels and infiltration of IgG4-positive plasma cells [1]. Subsequently, it was reported that several patients had developed mass-forming lesions in organs other than the pancreas. Thus, a novel disease entity was proposed and termed, 'IgG4-related systemic disease'. IgG is an immunoglobulin consisting of four subclasses (IgG1, IgG2,

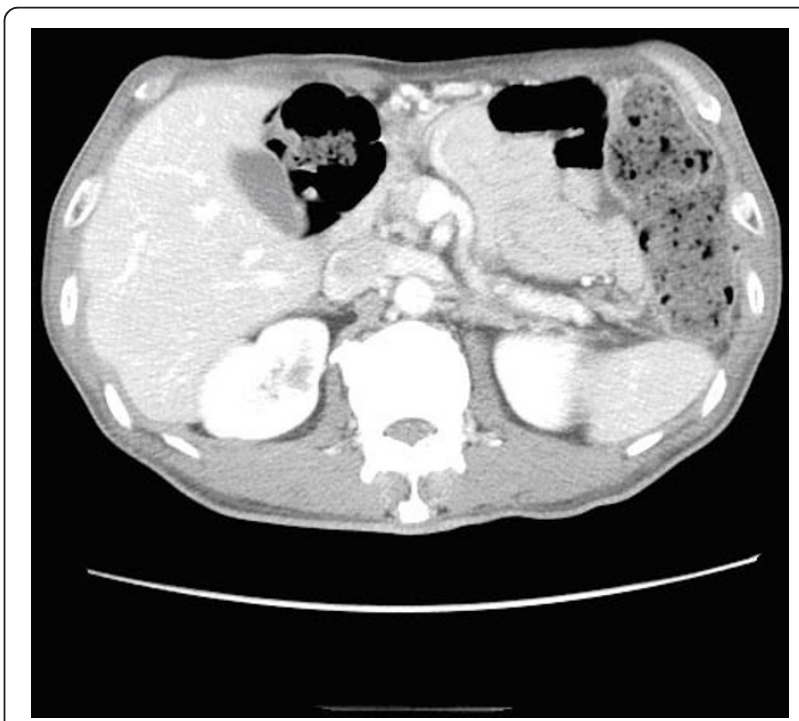

Figure $3 \mathrm{CT}$ showed reduction of the right renal mass, and disappearance of the left renal mass. 
IgG3, and IgG4). IgG4 is the rarest IgG subclass in the serum, and accounts for $6 \%$ or less of total IgG. Zen et al. reported that Th2 cells are predominant in lesions of IgG4-related disease that are associated with an infiltration of numerous CD4- and CD25-positive regulatory $\mathrm{T}$ cells (Tregs). Furthermore, the authors found that interleukin-10 and tumor growth factor- $\beta$ secreted from Tregs are involved in the proliferation of IgG4-positive plasma cells, excess IgG4 secretion, fibrosis and tumor formation [2].

IgG4-related systemic disease occurs more frequently in middle-aged and older men, with a male to female ratio of 2:1. The most common sites of occurrence are the pancreas and lung, but it can also occur in other tissues and organs including the common bile duct, salivary glands, kidney, prostate and retroperitoneum. In the kidney, IgG4-related disease sometimes manifests itself as tubulointerstitial nephritis, in which tumor formation is not a feature [3]. A definitive diagnosis of these diseases requires histopathological evidence of inflammatory cell infiltration, particularly IgG4-positive plasma cells. Lynn et al. proposed that IgG4-related disease should be definitively diagnosed based on the presence of at least 30 cells/HPF of IgG4-positive plasma cells in a lesion [4]. IgG4-related diseases also involve the formation of masses that are characterized by marked fibrosis and obliterating phlebitis.

It is important to differentiate these diseases from malignant tumors. To this end, for the diagnosis of autoimmune pancreatitis, the clinical diagnostic criteria were revised in 2006 to include high IgG4 levels and a mass-forming lesion outside the pancreas [5]. There are no established guidelines regarding the treatment of IgG4-related systemic diseases, so we based our treatment on the Japanese consensus guidelines for the management of autoimmune pancreatitis, as recommended by the Japan Pancreas Society. Treatment was initiated with $0.6 \mathrm{~kg} / \mathrm{mg} /$ day prednisolone, and the dose was tapered to $5 \mathrm{mg}$ every one to two weeks according to the clinical symptoms and results upon examination or imaging. To prevent recurrence, maintenance doses of 5 $\mathrm{mg}$ were required for three years [6].

The renal and pulmonary masses in our patient were suspected to be bilateral renal cancer and multiple lung metastases. However, inflammatory pseudotumors could not be ruled out, because our patient also had autoimmune pancreatitis and high serum IgG and IgG4 levels. Therefore, a CT-guided biopsy and pathological examination were performed, leading to the diagnosis of IgG4-related disease. There are some reports of patients who presented with a renal mass, and thus underwent a nephrectomy as the mass was mistaken for a malignant tumor. Many of these patients were subsequently diagnosed with IgG4-related systemic disease by pathological examination $[7,8]$. Since those patients did not have any clear symptoms aside from a renal mass, it seemed impossible to suspect IgG4-related disease based solely on imaging results. One reason for this may be that neither a definitive description of IgG4-related disease nor diagnostic criteria have been established. However, there are some types of prostatitis, idiopathic retroperitoneal fibrosis and renal masses that have been characterized as IgG4-related systemic diseases, and which are familiar to urologists.

\section{Conclusion}

We have presented a case of IgG4-related systemic disease characterized by masses in the kidney and lung, and diagnosed by CT-guided biopsy. Renal masses in particular require comprehensive diagnosis so that unnecessary surgical resection can be avoided. Further emphasis should be placed on the study of IgG4-related diseases in the future.

\section{Consent}

Written informed consent was obtained from the patient for publication of this case report and any accompanying images. A copy of the written consent is available for review by the Editor-in-Chief of this journal.

\section{Authors' contributions}

GN drafted the report. TY, YK, RK, KZ, MT, SA and TT cared for the patient. $\mathrm{YY}$ and $\mathrm{NH}$ reviewed the report. $\mathrm{KN}$ drafted the report and cared for the patient. All authors read and approved the final version of the manuscript.

\section{Competing interests}

The authors declare that they have no competing interests.

Received: 15 February 2011 Accepted: 25 September 2011 Published: 25 September 2011

\section{References}

1. Hamano H, Kawa S, Horiuchi A, Unno H, Furuya N, Akamatsu T, Fukushima M, Nikaido T, Nakayama K, Usuda N, Kiyosawa K: High serum lgG4 concentrations in patient with sclerosing pancreatitis. N Engl J Med 2001, 344:732-738.

2. Zen Y, Fujii T, Harada K, Kawano M, Yamada K, Takahira M, Nakanuma Y: Hepatology 2007, 45:1538-1546.

3. Saeki T, Nishi S, Ito T, Yamazaki H, Miyamura S, Emura I, Imai N, Ueno M, Saito A, Gejyo F: Renal lesion in IgG4-related systemic disease. Intern Med 2007, 46:1365-1371.

4. Cornell LD, Chicano SL, Deshpande V, Collins AB, Selig MK, Lauwers GY, Barisoni L, Colvin RB: Pseudotumors due to lgG4 immune-complex tubulointerstitial nephritis associated with autoimmune pancreatocentric disease. Am J Surg Pathol 2007, 31(10):1586-1597.

5. Okazaki K, Kawa S, Kamisawa T, Naruse S, Tanaka S, Nishimori I, Ohara H, Ito T, Kiriyama S, Inui K, Shimosegawa T, Koizumi M, Suda K, Shiratori K, Yamaguchi K, Yamaguchi T, Sugiyama M, Otsuki M, Research Committee of Intractable Diseases of the Pancreas: Clinical diagnostic criteria of autoimmune pancreatitis: revised proposal. J Gastroenterol 2006, 41(7):626-631.

6. Kamisawa T, Okazaki K, Kawa S, Shimosegawa T, Tanaka M, Research Committee for Intractable Pancreatic Disease and Japan Pancreas Society: Japanese consensus guideline for management of autoimmune pancreatitis: III. Treatment and prognosis of AIP. J Gastroenterol 2010, 45:471-477. 
7. Miyazaki C, Haratake J, Takano N, Sakino I, Nakamura N: Perirenal tumefactive IgG4-related disease without fibrosclerotic change. Jpn J Diagnostic Pathol 2009, 26(2):111-114.

8. Shoji S, Nakano M, Usui Y: IgG4-related inflammatory pseudotumor of the kidney. Intern J Urol 2010, 17:387-388.

\section{doi:10.1186/1752-1947-5-480}

Cite this article as: Nishikawa et al.: Inflammatory pseudotumors of the kidney and the lung presenting as immunoglobulin G4-related disease: a case report. Journal of Medical Case Reports 2011 5:480.

Submit your next manuscript to BioMed Central and take full advantage of:

- Convenient online submission

- Thorough peer review

- No space constraints or color figure charges

- Immediate publication on acceptance

- Inclusion in PubMed, CAS, Scopus and Google Scholar

- Research which is freely available for redistribution

Submit your manuscript at www.biomedcentral.com/submit
() Biomed Central 